\title{
La relación entre los estilos de aprendizaje y el uso de las tecnologías de información y comunicación en educación de personas adultas
}

\author{
The Relationship between Learning Styles and Use of Information and Communication \\ Technologies in Adult Education
}

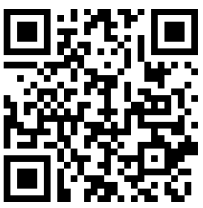

\author{
Lorena Acosta-Castillo ${ }^{1}$ \\ Corporación Universitaria Minuto de Dios \\ Bogotá, Colombia \\ lorenaa1980@hotmail.com
}

Recibido 23 de abril de 2015 • Corregido 7 de julio de 2016 • Aceptado 16 de agosto de 2016

\begin{abstract}
Resumen. El presente estudio tuvo como objetivo identificar los estilos de aprendizaje y las estrategias que usan aprendices de la población adulta, ante el uso de las TIC. Para lograrlo, se empleó un enfoque de investigación de corte cualitativo. Los participantes fueron personas de la comunidad que asisten a un proyecto denominado Alfabetización en el Manejo de la Información de una Corporación Universitaria. El grupo estuvo conformado por 100 personas, sus edades oscilaron entre los 40 y 59 años. Los instrumentos que se aplicaron fueron el inventario VARK para estilos de aprendizaje, la técnica de observación y el diario de campo. El inventario VARK permitió identificar los estilos de aprendizaje que emplean este estudiantado (la técnica de observación consistió en conocer el contexto en el cual se desarrolla, sus relaciones entre pares, relación con el personal docente) y analizar las estrategias empleadas para desarrollar los temas al integrar las TIC. Uno de los principales hallazgos que se obtuvo fue que el $70 \%$ presenta un estilo de aprendizaje multimodal, es decir, procesan el conocimiento en más de una forma.
\end{abstract}

Palabras claves. Personas adultas, personas adultas mayores, aprendizaje, educación, multimodal, TIC.

Abstract. This research aimed to identify the learning styles and the strategies used by adult students to involve ICTs in their knowledge. To achieve this, a qualitative research approach was carried out. The participants were members of the community who participated in a project called "Literacy in the Information Management of a University Corporation". The group consisted of 100 persons; their ages ranged between 40 and 59 years. The instruments used were the VARK inventory for learning styles, Observation and a field journal. The VARK inventory identified the learning styles used by adults. The Observation allowed the understanding of the environment or the context of these students, their relations with peers and with their teachers, and also to analyze the strategies used to develop topics through TICs integration. One of the main findings obtained was that $70 \%$ of the adult students have a multimodal learning style; for instance, they process knowledge in more than one way.

Keywords. Adults, elder, learning, education, multimodal, ICT.

\footnotetext{
${ }^{1}$ Originaria de Bogotá, Colombia. Realizó sus estudios profesionales en Trabajo Social en la Universidad Colegio Mayor de Cundinamarca y Especialización en Diseño de proyectos en la Universidad Internacional Iberoamericana en Colombia. Su experiencia de trabajo ha girado en torno al campo de la docencia a nivel de educación superior desde hace 7 años en la Corporación Universitaria Minuto de Dios; aquí ha desempeñando funciones como coordinadora de Práctica en Responsabilidad Social, espacio en el que se ofrecen y desarrollan procesos de formación básica en informática y programas de Office con el componente de formación ciudadana a personas que se encuentran en situación de vulnerabilidad y que generalmente no acceden fácilmente a este tipo de información; así mismo se desempeña como docente de la cátedra transversal de Emprendimiento y como formuladora de proyectos de ciencia, tecnología e innovación desde la plataforma del Parque Científico de Innovación Social.
} 
doi: http://dx.doi.org/10.15359/ree.20-3.10

URL: http://www.una.ac.cr/educare

CORREO: educare@una.cr

Al hablar de estilo de aprendizaje, se hace referencia a la manera que cada persona emplea para aprender; esta manera implica la utilización de algún método o estrategia que le facilita el aprendizaje, como es el escribir, el observar o el escuchar.

A las generaciones adultas que se incorporan hoy al espacio académico a través de las tecnologías de la información y la comunicación (TIC), les resultan difíciles ciertos procesos, debido a su historia de vida y estrategias empleadas en la escuela tradicional. Teniendo en cuenta la nueva sociedad de la información, las personas adultas sienten la necesidad de realizar cursos que les permitan ir a la vanguardia de las competencias digitales, por lo que tienen un interés por aprender en el menor tiempo posible. En este contexto, el profesorado que asume el reto de educar a personas adultas mayores en cuanto al manejo de las TIC debe tener en cuenta que este grupo de personas tiene "estilos de aprendizaje" distintos, por lo que sus docentes deben utilizar estrategias especiales orientadas a involucrarlas, sin olvidar sus temores y resistencias, al enfrentarse al manejo de nuevas herramientas tecnológicas.

\section{Antecedentes}

La incorporación de las TIC, hoy, se ve como la oportunidad para mejorar la calidad de vida de las personas; pero así mismo es necesario tener en cuenta el gran avance que estas han tenido y la apropiación de estas por las generaciones más jóvenes, llevando al nacimiento de una gran problemática a nivel social, la cual es el principio de una brecha digital, debido al limitado acceso, uso y manejo de algunos grupos sociales y etéreos como son las personas adultas y las personas adultas mayores.

La sociedad actual ha presentado grandes cambios derivados de la aparición de las TIC, tal como lo relatan diferentes estudios (Cabero, 2001; Castells, 2001; Elboj, Puigdellívol, Soler y Valls, 2003) que han tratado de exponer esta realidad en todos los ámbitos que supone la entrada y el desarrollo de la sociedad de la información. Coinciden en que se deben identificar las capacidades intelectuales y los recursos tecnológicos necesarios para el procedimiento de la información como factores claves de la actual sociedad.

El impacto de las TIC ha llegado a todas las esferas de nuestras vidas, incidiendo en los diversos grupos sociales, independientemente de la edad o idioma. El acceso a estas herramientas no se ha producido de una manera igualitaria. Lo anterior presume, para las personas que no pueden acceder a las TIC, un acrecentamiento de las desigualdades y una causa de exclusión social y cultural, desencadenando lo que se ha llamado hoy "brecha digital".

Los avances tecnológicos han llevado a las personas adultas que no manejan estas herramientas a conformar un grupo denominado "personas analfabetas digitales", quienes quedan limitadas a la hora de poder desarrollar actividades cotidianas con las TIC o cualquier innovación tecnológica destinada al uso habitual. 
De acuerdo con los múltiples cambios que se han dado por el desarrollo tecnológico en las últimas décadas, el sistema educativo se ha visto en la necesidad de replantear sus programas, indagando en nuevas estrategias que le permitan al individuo no solo ir al ritmo impuesto, sino también facilitarle la preparación y superación continua a lo largo de toda su existencia. Teniendo en cuenta lo anterior, la educación andragógica vincula el uso de las herramientas tecnológicas con el fin de permitir a sus participantes en edad adulta no solo la eliminación de la barrera o inhibición al uso de las TIC, sino su empleo para apropiarse del conocimiento que construyen y disminuir la brecha tecnológica existente entre la población jóvenes y la adulta.

Por tanto, se afirma que, si bien los sujetos adultos afrontan los desafíos que la nueva sociedad plantea, la realidad muestra que los procesos de integración, en cuanto al uso y apropiación de la tecnología, aún no están completamente adecuados. Por esta razón, surge este estudio en el cual se busca identificar cuáles estrategias emplean aprendices de edad adulta, de acuerdo con sus estilos de aprendizaje ante el uso de las TIC frente a los retos sociales y laborales que enfrentan. El fundamento teórico del estudio está en los denominados "estilos de aprendizaje" y en el modelo VARK.

\section{Marco teórico}

\section{Estilos de aprendizaje}

Uno de los temas que se ha venido fortaleciendo en esta nueva década en el área educativa ha sido la incorporación de las TIC con los estilos de aprendizaje, tal como lo afirman Torres y Redondo (2004):

La educación no ha sido ajena a ninguno de los procesos de convergencia tecnológica con influencias sociales, políticas y económicas; por esto, la tecnología está modificando significativamente los paradigmas educativos convencionales y creando nuevos modelos de enseñanza y aprendizaje basados en dimensiones multiculturales, y caracterizados en algunos casos por la desaparición de barreras como espacio y tiempo. (p. 11)

Esto permite generar mayor conectividad tecnológica entre las personas y acotación en los tiempos para la realización de diferentes procesos, lo cual se ve reflejado en el aprovechamiento del tiempo y una mayor relación entre las personas por medio de diferentes plataformas tecnológicas virtuales.

La dinámica de la sociedad que hoy gira en torno a las TIC, ello le exige a docentes y estudiantes que tengan los conocimientos básicos en cuanto al manejo e incorporación de estas en los procesos de enseñanza/aprendizaje, ya que esto les permite potencializar habilidades para crear estrategias y herramientas que les lleven a desarrollar competencias comunicativas, cognitivas e informáticas que les faciliten la interacción con el otro o la otra. 
doi: http://dx.doi.org/10.15359/ree.20-3.10

URL: http://www.una.ac.cr/educare

CORREO: educare@una.cr

Para plantear el significado de estilos de aprendizaje, iniciaremos por qué es aprendizaje. Feldman (2005, citado por Zapata-Ros, 2015) considera "el aprendizaje como un proceso de cambio relativamente permanente en el comportamiento de una persona generado por la experiencia" (p. 73); lo cual supone que existan cambios en la conducta del individuo que pueden ser perdurables en el tiempo. Según Schmeck (1988, citado por Zapata-Ros, 2015), “... el aprendizaje es un sub-producto del pensamiento... Aprendemos pensando, y la calidad del resultado de aprendizaje está determinada por la calidad de nuestros pensamientos" (pp. 73-74).

Hablando específicamente de los estilos de aprendizaje, Crispín, Esquivel, Loyola y Fregoso (2011) hacen énfasis en clarificar de dónde parten los distintos estilos de aprendizaje, desde la fuente fisiológica hasta las características especificas de la personalidad, identificando el sentido de pertenencia, el cual hace evidente estos estilos y el comprender cómo una persona logra pensar, aprender, enseñar o conversar de maneras diversas; esto, desde los aspectos cognitivos y procesos educativos.

Para Lozano (2009), los estilos de aprendizaje implican preferencias, tendencias y disposiciones que distinguen a un individuo en la manera en que se conduce, habla, piensa, aprende y enseña. Prado (2004) describe que las teorías de aprendizaje se han convertido en una alternativa para explicar el motivo por el cual un grupo de estudiantes que comparte el mismo espacio de aprendizaje aprende de diferentes maneras. Para Aguilera y Ortiz (2010), el perfeccionamiento de los estilos de aprendizaje en el estudiantado facilita el acceso a mejor información, adquisición de nuevos conocimientos y orientación sobre su sentido personal y capacidad creativa para la resolución de problemas con los cuales debe enfrentarse diariamente en su sociedad. Por otro lado, Contreras y Lozano (2012) hacen referencia a que el alumnado es "responsable de su aprendizaje y por lo tanto hablar de estilos de aprendizaje sin relacionar la característica de auto-regulación que tiene el propio estudiante, es como hablar de poseer una característica que igual da que la tenga (o no la tenga) si no es aplicada, mejorada y adaptada por el propio estudiante con su voluntad, compromiso, responsabilidad y ahínco" (p. 5).

\section{Las TIC en los procesos de enseñanza y aprendizaje}

Las TIC son un conjunto de medios o herramientas tecnológicas de la informática y la comunicación ... que podemos utilizar en pro del aprendizaje. [Estas han traído consigo] la facilidad de crear, procesar, difundir información ha roto todas las barreras que limita la adquisición del conocimiento, contribuyendo al desarrollo de habilidades y destrezas comunicativas entre docentes y estudiantes. (Rodríguez, 2009, Párr. 1)

Las TIC se han convertido en una de las herramientas básicas de la sociedad, por lo cual se ha hecho necesario ofrecer a la ciudadanía una educación que tenga en cuenta una nueva dinámica en donde las oportunidades educativas han de ser consideradas en dos aspectos: en 
su conocimiento y en su uso. El primer aspecto, el conocimiento, es consecuencia directa de los cambios de cultura de la sociedad actual, en donde no se puede entender el mundo de hoy sin un mínimo de cultura informática. Esto evidencia la necesidad de entender cómo se accede a la información y cómo se transmite en sus múltiples manifestaciones, ya sea a través de textos, imágenes, sonidos, diagramas. El segundo aspecto, el uso, se halla muy conexo con el conocimiento, pero de una manera más técnica, ya que hace referencia a que se deben emplear las TIC para aprender y para enseñar. Entendiéndose que la enseñanza y el aprendizaje de cualquier materia o habilidad se puede hacer de una manera más fácil mediante el empleo o incorporación de las TIC.

El uso de las TIC ha innovando la educación intensamente, debido a que han llevado a cambiar tanto la forma de enseñar como la forma de aprender, en donde el rol docente y estudiantil se ha ido modificando, así como los objetivos formativos que no solo van en dirección de aprendizajes de contenidos sino también en dirección a la formación para aprender a utilizar e incorporar estos nuevos medios para producir conocimiento. La incorporación de las TIC en los procesos de enseñanza aprendizaje motiva al personal docente a realizar procesos concienzudos de cambio de estrategias de comunicación con sus estudiantes, asumiendo un rol de facilitador y mediador del aprendizaje, con el fin de orientarles hacia la planificación y alcance de los objetivos. Los cuerpos docentes no deben olvidar que las TIC hoy presentan un gran apoyo para la generación de conocimiento y apropiación de este mismo, al generar diferentes recursos como son los entornos virtuales, internet, blogs, wikis, webquest, foros, chat, mensajerías, videoconferencias; que permiten el fortaleciendo del aprendizaje significativo, activo y flexible.

No obstante, de acuerdo con Rodríguez (2009), no podemos ignorar que estas nuevas herramientas tienen una gran importancia dentro de la nueva sociedad del conocimiento, que en consecuencia conllevan a que la educación se acomode a las exigencias que aún tiene respecto de esta. Para este nuevo reto de la educación, debemos reconocer que la escuela debe enfrentarse con altura a él y velar porque esta llegue con eficiencia y calidad a la usuaria y usuario indefenso y lleno de curiosidad frente al conocimiento e involucramiento de las nuevas herramientas tecnológicas en él.

\section{Características de las personas adultas en el contexto educativo}

Estudiantes de edad adulta se consideran como personas autónomas con capacidad de elegir responsablemente previniendo las consecuencias y posibles efectos de las decisiones por las que haya optado. Además, cuentan con la experiencia adquirida que influye definitivamente en sus futuros aprendizajes. Freire (2002) señala que la formación de la persona adulta puede y debe ser continua e inacabada, ya que el pensamiento es resultado del concepto de inacabamiento del ser humano y la consecuencia de ello es un sujeto discente consciente y autónomo, condicionado por sus aspectos genéticos, culturales y sociales. Así también, Adam 
doi: http://dx.doi.org/10.15359/ree.20-3.10

URL: http://www.una.ac.cr/educare

CORREO: educare@una.cr

(1987) señala que la persona adulta, como estudiante, presenta características peculiares y diferentes, por lo que precisa no solo de una metodología, sino de todo un sistema educativo que, fundamentado en principios filosóficos y psicosociales, responda al proceso de enseñanzaaprendizaje y de la formación de personas adultas.

Según Knowles, Swanson y Holton (2001), el sujeto adulto se caracteriza por la necesidad de conocer las motivaciones que justifican sus intenciones de estudiar; este se percibe a sí mismo como independiente y autónomo respecto de los demás. En la educación de personas adultas, la experiencia juega un papel muy importante, ya que con base en esta se fundamentan los nuevos aprendizajes o barreras que esta interponga para anclar o no su aprendizaje previo con el deseado.

El aprendizaje en la población adulta se ve influenciado por diversos factores y variables que configuran dicho proceso, lo cual lo hace diferencial del aprendizaje de los demás grupos. El estudiantado adulto, a medida que avanzan en su proceso de enseñanza-aprendizaje, tiende a configurar un estilo de pensamiento que lo caracteriza. No llega "en cero" a una situación de aprendizaje. En especial, si hacemos referencia a contextos de formación vinculadas a su trabajo, podemos decir que el sujeto adulto en rol de estudiante trae consigo un bagaje de experiencia laboral, de su vida personal y un trayecto por la educación formal.

\section{Metodología de investigación}

El enfoque de investigación seleccionado y abordado es de corte cualitativo, dado que ofrece gran flexibilidad en su aplicación, así como una gran variedad de instrumentos, los cuales permiten recoger datos de manera descriptiva y observar a los sujetos participantes en sus entornos naturales, con lo cual se logra una mejor comprensión del problema de investigación. Como lo plantean Hernández, Fernández y Baptista (2006), el propósito en la recolección de datos en el enfoque cualitativo no es medir variables para realizar análisis estadísticos; por el contrario, lo que se busca es obtener datos de los sujetos involucrados o contextos en sus propias formas de expresión. En este caso, se relaciona con la incorporación de las TIC en la manera como se deben emplear para que se mejoren los procesos de aprendizaje. Esta investigación se interesa más en saber cómo se establece la dinámica o cómo ocurre el proceso en que se da la incorporación de las TIC para mejorar los procesos de aprendizaje, pues el impacto de las TIC ha llegado a todas las esferas de nuestras vidas, y ha incidido en los diversos grupos sociales sin tener en cuenta la edad o idioma.

Lo anterior nos lleva a investigar de qué manera se puede optimizar el uso de las TIC para el mejoramiento de los procesos de aprendizaje, teniendo en cuenta los estilos de aprendizaje empleados por los sujetos adultos, para quienes las TIC se convierten en una herramienta necesaria. Los efectos de su uso en el ámbito educativo dependen del enfoque pedagógico, de los objetivos propuestos, la adaptación al contexto y características del estudiantado; como lo 
exponen Ferreiro y Napoli (2007) cuando afirman que lo importante no es simplemente tener tecnología, sino lo que importa es hacer un apropiado uso de esta moldeándola a los objetivos que se pretenden alcanzar. Por lo anterior, el enfoque cualitativo es el que orienta el proceso de esta investigación, puesto que se entrevistaron y observaron estudiantes en situaciones educativas mediadas con las TIC, con el objetivo de identificar las ventajas para quienes tienen estilos de aprendizaje diferentes a los que se utilizan en la enseñanza tradicional.

\section{Instrumentos de investigación}

Los instrumentos que se aplicaron, de acuerdo con las características y enfoque de esta investigación, fueron el inventario VARK para estilos de aprendizaje, la técnica de observación y el diario de campo. El inventario VARK fue utilizado para identificar los estilos de aprendizaje que emplean las personas adultas; mientras que los instrumentos de observación y diario de campo fueron aplicados para conocer el contexto del estudiantado, incluyendo aspectos como relaciones entre pares, relación con su docente y las estrategias empleadas para desarrollar los temas al integrar las TIC.

\section{El inventario VARK}

El modelo VARK (2013) fue creado por Neil Fleming y Colleen Mills; ellos propusieron que las personas reciben información a través de los sentidos, y el cerebro procesa y selecciona parte de esa información. Esta selección se realiza en función de los intereses de las personas y la forma en que es percibida. Este modelo toma el nombre de VARK, por las siglas en inglés de las modalidades sensoriales: visual, auditivo, leer y quinésico. Cada una de estas modalidades se describe por características esenciales de la forma como el alumnado percibe la información y la procesan, según sus intereses

El inventario está compuesto por un cuestionario que consta de 16 preguntas; cada una de estas, a su vez, tiene 4 opciones de respuesta que corresponden a cada uno de los estilos de aprendizaje. Para dar solución al cuestionario, la persona debe seleccionar la respuesta que mejor explique su preferencia, y marcar la letra que represente su elección; si siente que alguna de las respuestas que están allí no se relacionan con su actuar, la debe dejar en blanco y al menos debe contestar 12 de las 16 peguntas del cuestionario.

\section{La observación}

Se empleó la observación directa no-participante. Con esta técnica se tuvo riguroso cuidado en ir anotando todo lo que se observaba, ya que un dato puede ser el anclaje de otro. En esta investigación, la observación tuvo como propósito recoger evidencias acerca de los 
doi: http://dx.doi.org/10.15359/ree.20-3.10

URL: http://www.una.ac.cr/educare

CORREO: educare@una.cr

aspectos involucrados en el proceso de enseñanza y aprendizaje tanto del personal docente como de sus estudiantes. La observación realizada de aula fue más que una percepción, ya que se encontraba dirigida a un objetivo específico y una población determinada, en este caso, estudiantes de edad adulta del proyecto de Alfabetización en el Manejo de la Información.

El instrumento de observación constó de 26 aspectos a observar, divididos en tres momentos de la sesión como es el inicio, el desarrollo y el cierre; cada uno de estos momentos cuenta con unas preguntas, las cuales van orientando hacia lo importante de la observación.

\section{Diario del investigador o investigadora}

El instrumento diario del investigador o investigadora, según Porlán (1987), es una herramienta para la reflexión significativa y vivencial de profesionales del campo educativo, un instrumento básico para la investigación en el aula, ya que puede adaptarse, por su carácter personal, a todo tipo de circunstancias. Latorre (1996) lo define como un instrumento de formación, que facilita la implicación y desarrolla la introspección; y de investigación, que desarrolla la observación y la auto observación recogiendo observaciones de diferente índole. Torres (1986) considera que el diario no solo es un elemento primordial para conocer lo que sucede en las aulas, sino que también es un instrumento adecuado para la investigación del propio profesorado.

Este instrumento en la investigación se aplicó durante el proceso de identificación del problema y la aplicación del inventario de estilos y la observación. En el diario se fue consignando en detalle cada uno de los acontecimientos que se identificaron en el trabajo de campo, esto permitió recordar con claridad los hechos en el momento de realizar el análisis de los datos y así mismo acumular un material de observaciones in situ que permitiera, con posterioridad, elegir los datos utilizados para dar a conocer los resultados de la investigación; de igual forma, el diario de campo permitió ir registrando las experiencias de los sujetos adultos en el proceso de aprendizaje en el empleo de las TIC, y las afirmaciones o comentarios de estos en el momento de la realización de algún ejercicio o tarea direccionada por su docente.

\section{Procedimiento de la investigación}

El procedimiento llevado a cabo para la investigación se desarrolló en cuatro etapas. La primera correspondió al diseño de los instrumentos de investigación para la recolección de datos consistentes en la observación. En la segunda se desarrolló una reunión de preparación con el docente encargado del proyecto, en la cual se le dio a conocer el objetivo de la investigación, el modelo que la fundamenta (VARK), los instrumentos a utilizar (incluyendo el instrumento de observación no participante) y los momentos en los cuales se aplicarían estos instrumentos. La 
tercera etapa correspondió a la aplicación de los instrumentos de recolección de la información; para la aplicación del instrumento se realizó una breve explicación de la actividad al grupo de estudiantes, se le informó el objetivo a alcanzar y la necesidad de contestar la encuesta de manera sincera. Otro momento de la etapa se dio con la técnica de observación directa diligenciando el instrumento diseñado. La cuarta y última consistió en la sistematización y análisis de los datos recolectados a través de los instrumentos, con el fin de darle respuesta a la pregunta de investigación planteada.

\section{Estrategia de análisis de datos}

Una vez realizada la aplicación del inventario VARK, efectuada la observación y diligenciamiento del diario de campo, se revisó todo el material en su forma original y se organizaron los datos por cada participante de forma independiente. Posterior a esto se realizó el proceso de codificación, mediante una lectura detallada de las respuestas estudiantiles para iniciar "el proceso de identificación de palabras, frases, temas o conceptos dentro de los datos de manera tal que los patrones subyacentes pudieran ser identificados y analizados" (Morse \& Field, 1995, en Mayan, 2001, p. 24). Posterior a esto se analizaron segmentos de texto cuestionando su significado por medio de un proceso de comparación constante, se indujeron categorías con los segmentos similares que luego fueron vertidos a una tabla hasta completarla, de donde emergieron las categorías de análisis.

Posterior a esto se realizó una triangulación de datos, donde se confrontaron los datos recabados con la aplicación del inventario VARK, los datos obtenidos al realizar la observación de las sesiones de clase y, por último, el cruce de información con investigaciones relacionadas con los estilos de aprendizaje de los sujetos adultos.

\section{Resultados}

Los resultados aquí mostrados nos permiten dar respuesta a la pregunta de investigación, con base en la relación que puede establecerse entre las tecnologías de la información y comunicación y el proceso de educación de personas adultas, considerando sus estilos de aprendizaje según la teoría de estilos de aprendizaje de Fleming.

\section{Resultados del instrumento de estilos de aprendizaje}

En cuanto a los estilos de aprendizaje, se encontró que el 70\% (figura 1) de las personas adultas tienen un estilo multimodal, lo que quiere decir que para su aprendizaje procesan la información en más de una forma, lo que les puede llevar más tiempo para reunir información. Esto les lleva a tener una comprensión más amplia y profunda de los temas. Este resultado tiene una gran similitud con pruebas realizadas con muestras grandes; es el caso de Fleming, quien 
doi: http://dx.doi.org/10.15359/ree.20-3.10

URL: http://www.una.ac.cr/educare

CORREO: educare@una.cr

tiene los resultados de una muestra de 31,243 personas, en donde las multimodales $\mathrm{m}$ fueron un $58 \%$, los quinestésicos $\mathrm{k}$ formaron un $18 \%$, las personas con preferencia de lectura y escritura $\mathrm{R}$ un $16 \%$, las auditivas a un $5 \%$ y las visuales v un $3 \%$.

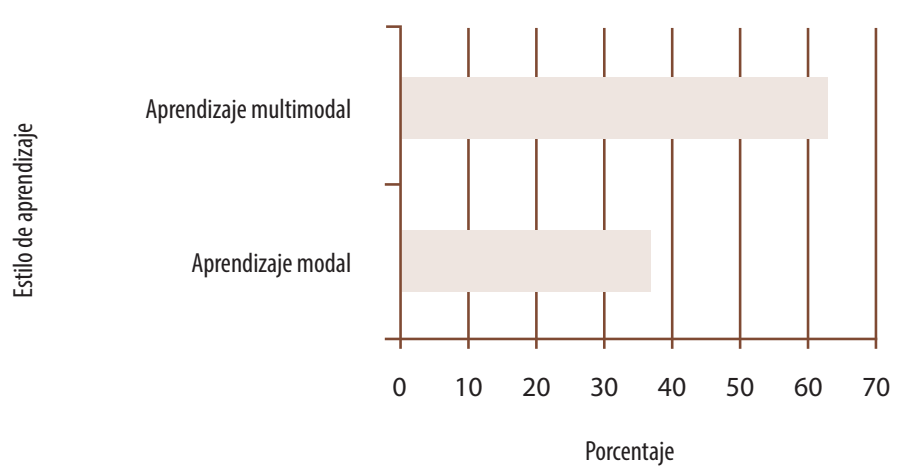

Figura 1. Estilo de aprendizaje de las personas adultas mayores.

En relación con las combinaciones de aprendizaje multimodal, podemos encontrar que, en los sujetos adultos, el estudio evidenció que las siguientes combinaciones de aprendizaje se presentan en un mayor porcentaje (figura 2):

- La combinación de estilos, visual y qinestésico, ocurre en un 16\%; esta combinación corresponde a las personas que aprenden principalmente mediante imágenes, se fijan en las características que pueden ver e imaginar y al mismo tempo aprenden mediante el tacto, el movimiento y las percepciones sensoriales.

- La combinación de estilos visual y lectura/escritura se presenta en un $11 \%$, con personas que, aparte de involucrar imágenes para su aprendizaje, aprenden mejor cuando reciben y devuelven la información en palabras, en donde la comunicación puede ser por escrito o tecleada y las herramientas de elección son los diccionarios, el internet, respuestas escritas y signos de texto.

- La combinación de estilos visual y auditivo sucedió en un $10 \%$ de los casos, en personas que se apoyan en imágenes, pero al mismo tiempo aprenden las lecciones como secuencias memorizadas casi de forma literal; son personas que necesitan el silencio para estudiar y preferirán escuchar los temas o que se los lean. 


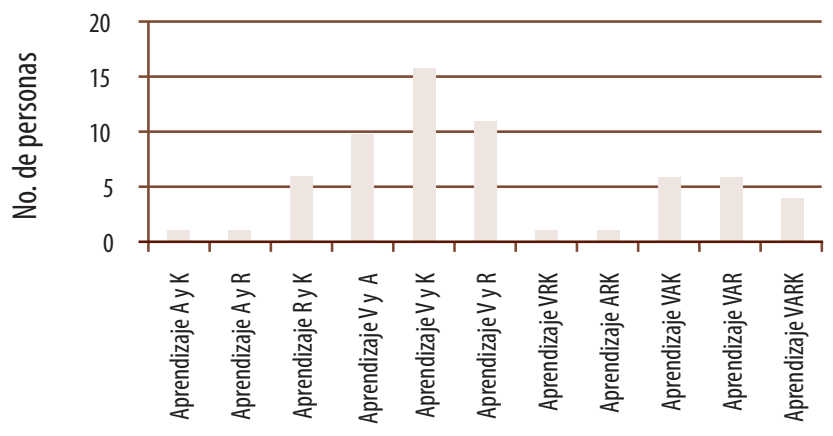

Estilos de prendizaje

Figura 2. Aprendizaje multimodal.

De acuerdo con lo anterior, se evidencia que prevalece, entre estas combinaciones, el aprendizaje visual mezclado con algún otro estilo de aprendizaje. Este estilo de aprendizaje emplea un conjunto de organizadores gráficos que permiten ayudar al estudiantado, mediante el trabajo con ideas y conceptos, a pensar y a aprender más efectivamente. Este tipo de aprendizaje les permite identificar ideas erróneas y visualizar patrones e interacciones en la información, factores necesarios para la comprensión e interiorización de los conceptos.

En cuanto al aprendizaje modal (figura 3), el estudio evidenció que el modo de más relevancia es el aprendizaje visual, dado que la gente prefiere usar colores, imágenes, mapas, dibujos y gráficos para comunicar y organizar la información. La investigación también indicó que el modo que le sigue en el orden de relevancia es el estilo de aprendizaje quinestésico, lo que corresponde a que los sujetos adultos aprenden mejor haciendo, involucrándose y participando activamente en su proceso de aprendizaje, tanto con movimientos finos como con movimientos que involucran todo su cuerpo. En el trabajo se encontró que los estilos que tienen la menor relevancia son el estilo de aprendizaje -lecto-escritor-read/write, que corresponde a los adultos que aprenden mejor cuando reciben y devuelven la información en palabras y, en el último lugar, el estilo de aprendizaje auditivo, que caracteriza a las personas cuyo aprendizaje depende de escuchar y hablar. 
doi: http://dx.doi.org/10.15359/ree.20-3.10

URL: http://www.una.ac.cr/educare

CORREO: educare@una.cr

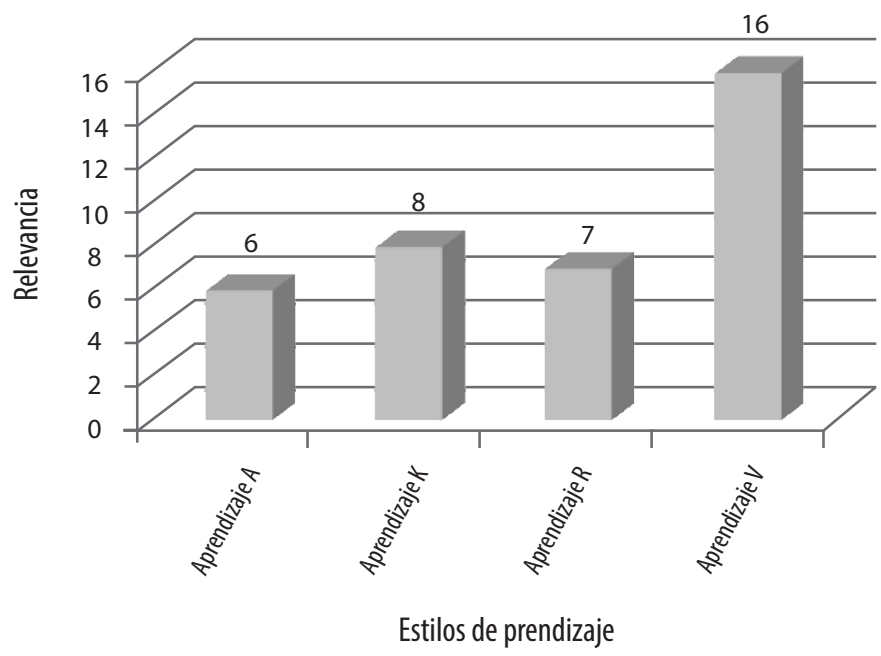

Figura 3. Aprendizaje modal.

\section{Resultados de la observación y diario de campo}

Se observó que existe un buen nivel de motivación y participación en las diferentes actividades por parte de las personas adultas, las cuales se caracterizan por la espontaneidad, la satisfacción ante la asignación de tareas que se ejecutan con gran sentido de responsabilidad y autonomía. Se evidencia un buen nivel de comunicación con su docente, lo cual se vio reflejado en las adecuadas relaciones interpersonales, al realizar un adecuado uso del lenguaje espontáneo y amplio vocabulario. Aparecen reflexiones adecuadas ante la toma de decisiones. Se observa que aprenden de una manera más reflexiva y persistente ante la ejecución de tareas. Los sujetos adultos logran un adecuado nivel de concentración en las actividades, con un buen empleo de nexos lógico-abstracto y generalizaciones adecuadas.

En una de las sesiones, la profesora trabajó el tema del uso del procesador de texto Word. Para esto, la docente realizó la explicación sin emplear material visual alguno, solamente hablando, mientras que algunos o algunas estudiantes iban buscando en el monitor los iconos que les decía, y otra parte anotaba en sus cuadernos las indicaciones entregadas. Como respuesta a esta situación, se pudo observar la expresión de frustración en los sujetos adultos ante la dificultad para comprender las instrucciones a seguir, así como para ubicar las herramientas en el monitor que les indicaba la docente. A partir de esta observación, se pudo establecer que su estilo aprendizaje no es el auditivo, ya que la metodología empleada por la profesora fue simplemente explicativa, y no comprendieron el tema; un grupo pequeño tomó apuntes del tema, otros grupos no encontraron interés en el tema por falta de motivación y de lograr captar la atención por parte de la profesora, lo que les llevó a aprender por sí en el computador e ir indagando sobre el tema. 
En otra sesión se contó con la participación de las mismas personas de la clase anterior, pero en diferente sala de sistemas, se observaron expresiones gestuales de inconformismo; al ingresar a la nueva sala de sistemas se expresan motivación, ya que es más amplia, tienen computadoras más modernas, hay ventilación, está alejada del ruido de la calle y hay video beam; la profesora inició preguntando sobre las dudas que les quedaron con el tema abordado la clase anterior, Word; el estudiantado manifiesta que no recuerda el tema. Frente a esto la profesora inicia la explicación, trae preparada una presentación en prezi; se motivan, toman apuntes y van haciendo el paso a paso en el computador, se observa que una de las personas adultas trae una grabadora de bolsillo y graba la clase a la par que va tomando apuntes en su cuaderno; sin embargo, manifiestan la desventaja que ven frente porque las computadoras son más "modernas", ya que no tienen un conocimiento de esa nueva versión. De esta clase se puede extraer que el ambiente influye en el aprendizaje, ya que genera estimulación visual, organización y una sensación de acogida y seguridad. Así mismo se ve que las personas adultas emplean diferentes estilos de aprendizaje al tiempo; de lo que se concluye que su estilo de aprendizaje es multimodal.

En la siguiente sesión, se explica power point; la profesora planteó las siguientes indicaciones "nadie tomeel computador, no tomen apuntes, todos van a estar muy atentos a mi explicación", cuando finalizó dio un tema y explicó la actividad a realizar. Muy pocas personas entendieron la actividad. Aquí se analiza que las personas adultas que iniciaron la actividad sin inconveniente aplican un estilo de aprendizaje netamente visual, en comparación con quienes no pudieron realizar el ejercicio. Con el estudiantado que no logró dar inicio a la ejecución de la actividad, la profesora debió volver a realizar la explicación sin poner como condición el "no poder tomar apuntes". La gran mayoría, paralelamente, iban interactuando con el computador aplicando lo explicado por la profesora; en este ejercicio podemos ver la combinación del aprendizaje visual y quinestésico, en donde el interactuar a través de la participación activa con el computador les permite aprender acerca de ello.

En el último encuentro observado, se evidenció cómo las personas adultas mezclan el estilo de aprendizaje visual y auditivo, en el momento en que la profesora realiza la explicación de cómo crear un correo electrónico, algunas de las personas realizaron mapas mentales en donde plasmaron el procedimiento que habían entendido y posteriormente realizaron el ejercicio en el computador siguiendo el paso a paso de lo que habían anotando.

De acuerdo con el instrumento de observación, se puede decir que en el aprendizaje de las personas adultas influye de manera directa el clima del aula (ventilación, espacio, luz), el cual debe ser propicio, no debe ser solo visto como un contexto motivador adecuado para el aprendizaje. Otro factor a tener en cuenta es la experiencia, ya que la persona adulta está envuelta en algún tipo de aprendizaje a través de toda su vida que va repercutiendo en sus nuevos aprendizajes llevándoles a estar actualizados. El siguiente factor y el más importante es la motivación; en las actividades realizadas, la motivación fue un factor determinante, ya que, si se encontraban motivadas hacia el ejercicio, mejor eran los resultados. Esta motivación no 
doi: http://dx.doi.org/10.15359/ree.20-3.10

URL: http://www.una.ac.cr/educare

CORREO: educare@una.cr

solo se relaciona con la clase y el tema a desarrollar, sino también con el apoyo y estimulación que las familias o redes manifiesten a las personas, les hagan ver que lo que están haciendo es importante y no que simplemente se encuentran participando de un curso para ocupar su tiempo libre. Otro de los factores importantes observados fue el papel del profesorado, ya que se convierte en facilitador para el proceso de aprendizaje de las personas adultas y adultas mayores. Teniendo en cuenta que desde sus intervenciones, metodologías, lenguaje y confianza que genere, les va a permitir interactuar de una manera más cercana con las tecnologías y hacer este proceso de más tranquilo y con la plena certeza de que sí lograrán aprender.

El ejercicio de observación igualmente permitió ver que las razones por las que las personas mayores aprenden el uso de la computadora son diversas, como adquirir nuevos conocimientos y elevar la autoestima; otras responden a necesidades más concretas, como prepararse para ayudar a sus hijos e hijas y nietos o nietas en sus estudios; conseguir un nuevo empleo o mantenerse en el actual.

Se pudo verificar que las personas adultas y adultas mayores presentan diversos estilos de aprendizaje, como son visual, auditivo, quinestésico y lecto/escritory, más aún, en la incorporación de las TIC en su diario vivir. Se logró evidenciar que, debido a sus aprendizajes anteriores, el adaptarse al manejo de las TIC no es una tarea fácil, ya que su proceso cognitivo es más lento, lo cual les lleva a sentir frustración y desespero por no asimilar fácilmente los nuevos contenidos.

Uno de los objetivos trazados en esta investigación fue el identificar el impacto de las TIC en la vida cotidiana de las personas adultas y adultas mayores. En las clases observadas se pudo identificar la búsqueda de independencia de las personas adultas y las personas mayores de sus familias en relación con las TIC.

El segundo objetivo de estudio fue analizar los efectos que trae el no conocimiento de las tecnologías para el sujeto adulto mayor; como ya se dijo anteriormente, en el ejercicio de observación realizado se vio la necesidad que tiene por incorporar a sus actividades las TIC, darles un buen uso a estas, ya que si no las conocen o no las usan, esto les trae consecuencias negativas en su desarrollo social, emocional y personal, pues les genera la sensación de aislamiento, exclusión de los avances que va teniendo la sociedad y, así mismo, pueden perder oportunidades a nivel laboral.

El último objetivo a alcanzar era determinar los principales estilos de aprendizaje que emplea el sujeto adulto para aprender a interactuar con las nuevas tecnologías de la información y la comunicación, de acuerdo con el inventario VARK. Aquí se puede determinar que las personas adultas y adultas mayores no tienen una única manera de aprender, desarrollan diferentes estilos de aprendizaje cuando de incorporar cognitivamente nuevos temas se trata. Se presenta un estilo de aprendizaje multimodal, de una manera indirecta van desarrollando diferentes estrategias que les permiten la comprensión e interiorización de los temas. Dentro de este aprendizaje multimodal, vale la pena hacer mención al modelo de Kolb, el cual está muy extendido para el diagnóstico de los estilos de aprendizaje en niveles adultos. 
De acuerdo con Kolb (1976, citado por García, 2006, Los estilos de aprendizaje desde la perspectiva de David Kolb, Párrf. 1):

El aprendizaje comienza con una experiencia inmediata y concreta que sirve de base para la observación y la reflexión. Estas observaciones se integran en una "teoría" formando conceptos abstractos y permitiendo su generalización tras comprobar las implicaciones de los conceptos en situaciones nuevas. Estas implicaciones o hipótesis sirven de base para generar nuevas experiencias.

El profesorado y las metodologías son otro factor importante en el proceso de aprendizaje de las personas adultas en relación con las TIC, ya que se debe tener en cuenta cada una de las capacidades que tienen estas persoans, como son el involucramiento directo a experiencias nuevas, la reflexión acerca de estas experiencias y la observación desde múltiples perspectivas, el ser capaz de crear nuevos conceptos, entre otras.

El inventario VARK, la observación y el diario de campo nos permiten ver que los recursos de aprendizaje deben ser lo suficientemente variados como para dar cabida al amplio bagaje de experiencias y conocimientos de estas personas, así como a sus tipos o estilos de aprendizaje preferidos. En esta línea se debe proporcionar un amplio número de recursos que permitan un análisis de los problemas desde varias perspectivas: fuentes de información diversas, formas diferentes de representación de los contenidos (documentos, gráficos, vídeos, animaciones) y un lenguaje apropiado. Se debe tender a organizar la formación en torno a casos prácticos y experiencias relevantes, y seleccionar contenidos de aprendizaje que tengan utilidad para resolver los problemas reales con los que se encontrarán las personas adultas, lo que les proporcionará experiencias de aprendizaje ricas, diversas y contextualizadas.

Hay que recordar que la enseñanza de las personas mayores no se puede tratar del mismo modo que la enseñanza de la población infantil o adulta joven; primero, debido a que sus capacidades físicas y cognitivas han ido disminuyendo; y segundo, a que estas personas acudieron a procesos educativos en donde se empleaban diferentes métodos de enseñanza.

\section{Conclusiones}

De acuerdo con la investigación realizada para identificar los estilos de aprendizaje y las estrategias que usan aprendices de edad adulto ante el uso de las TIC, se encuentra como hallazgo que la mayoría (alrededor del 70\%) es multimodal, ya sea por la combinación de dos, tres o las cuatro preferencias. Lo anterior parece sugerir que las personas adultas ven las herramientas tecnológicas como factores claves para mejorar sus procesos de aprendizaje, que parten de sus experiencias para lograr encajar su nuevo aprendizaje en las actividades diarias y relacionar los temas con situaciones vividas en otros escenarios. Así mismo, estas combinaciones llevan a las personas adultas a ser capaces 
doi: http://dx.doi.org/10.15359/ree.20-3.10

URL: http://www.una.ac.cr/educare

CORREO: educare@una.cr

de involucrarse enteramente y sin perjuicios en las situaciones que se les presentan, reflexionando en torno a las experiencias y percibiendo, desde varios ángulos, los retos que deben asumir.

La instrucción de las personas adultas mayores no se puede impartir del mismo modo que a la niñez o juventud actual. El efecto generacional y la transformación que ha habido en la sociedad, como resultado de los avances en la ciencia y la tecnología, han propiciado cambios sustanciales en las nuevas generaciones. Por lo anterior, las capacidades físicas y cognitivas entre ambos grupos de aprendices son distintas. Un aspecto que se podría resaltar es que el impulso que han tenido los videojuegos en la última década ha propiciado que las nuevas generaciones tiendan a ser más visuales, mientras que las personas adultas mayores, que no tuvieron acceso a esas tecnologías, posean estilos multimodales debido quizá a las exigencias de su entorno y a la ausencia de esas tecnologías en sus edades tempranas.

Otro de los hallazgos fue constatar lo estipulado por la teoría de Fleming respecto a la necesidad de emplear una combinación de estrategias que puedan integrar con otro estilo adicional al que domina el estudiantado, ya que las personas adultas demostraron el empleo de diversas habilidades para poner en contexto sus aprendizajes, realizando relaciones de situaciones nuevas frente a situaciones de anterioridad.

Un nuevo hallazgo y algo que no se esperaba encontrar fue la inmensa necesidad manifiesta de las personas adultas mayores por aprender a interactuar a través de las TIC, ya que ven estas herramientas como la única manera en la cual las personas se puede comunicar hoy. Han tenido que cambiar su dinámica de vida para poder ser acogidos y acogidas por la nueva sociedad, en donde el emplear adecuadamente un celular, un computador, tener correo electrónico, les permitirá llevar una mejor relación con su núcleo familiar y, de igual manera, mejorar su calidad de vida.

\section{Referencias}

Adam, F. (1987). Andragogía. Ciencia de la educación de adultos (2a ed.). Caracas: Universidad Nacional Experimental Simón Rodríguez. Publicaciones de la Presidencia. Recuperado de http://www.scielo.org.ve/scielo.php?script=sci arttext\&pid=S1316-00872007000200008

Aguilera, E. y Ortiz, E. (2010). La caracterización de perfiles de estilos de aprendizaje en la educación superior, una visión integradora. Revista Estilos de Aprendizaje, 5(5), 1-20. Recuperado de http://www2.uned.es/revistaestilosdeaprendizaje/numero 5/articulos/ Isr 5 articulo 2.pdf

Cabero, J. (2001). Tecnología educativa. Diseño y utilización de medios en la enseñanza. Barcelona: Paidós.

Castells, M. (2001). Internet y la sociedad red. Recuperado de http://www.uoc.edu/web/cat/ articles/castells/castellsmain2.html 
Contreras, Y. I. y Lozano, A. (Octubre, 2012). Aprendizaje auto-regulado como competencia para el aprovechamiento de los estilos de aprendizaje en alumnos de educación superior. Revista Estilos de Aprendizaje, 10(10), 1-39. Recuperado de http://www2.uned.es/ revistaestilosdeaprendizaje/numero 10/articulos/Articulo09.pdf

Crispín, M. L., Esquivel, M., Loyola, M. y Fregoso A. (2011). ¿Qué es el aprendizaje y cómo aprendemos? En M. L. Crispín (Coord.), Aprendizaje autónomo: Orientaciones para la docencia (pp. 10-28). México: Universidad Iberoamericana. Recuperado de http:// docplayer.es/16373502-Aprendizaje-autonomo-o-r-i-e-n-t-a-c-i-o-n-e-s-p-a-r-a-l-a-d-o-ce-n-c-i-a.html

Elboj, C., Puigdellívol, I., Soler, M. y Valls, R. (2003). Comunidades de aprendizaje. Transformar la educación. Barcelona: Grado.

Ferreiro, R. F. y de Napoli, A. (2007). Más allá del salón de clases. Los nuevos ambientes de aprendizajes. Revista Complutense de Educación, 19(2), 336-346. Recuperado de https:// revistas.ucm.es/index.php/RCED/article/viewFile/RCED0808220333A/15480

Freire, P. (2002). Pedagogía del oprimido. Rio de Janeiro: Paz e Tierra.

García, J. L. (2006). Algunos modelos de estilos de aprendizaje. Recuperado de http://www.jlgcue. es/modelos.htm

Hernández, R., Fernández, C. y Baptista, M. (2006). Metodología de la investigación. México: McGraw-Hill.

Knowles, M. S. y Swanson, R. A. y Holton, F. H. (2001). Andragogía: El aprendizaje de los adultos. México: Oxford University Press.

Latorre, A. (1996). El diario como instrumento de reflexión del profesor novel. En Actas del III Congreso de E. F. de Facultades de Educación y XIV de Escuelas Universitarias de Magisterio. Guadalajara: Ed. Ferloprint.

Lozano, A. (2009). Estilos de aprendizaje y enseñanza: Un panorama a la estilística educativa. México: Trillas.

Mayan, M. J. (2001). Una introducción a los métodos cualitativos: Módulo para estudiantes profesionales (Trad. C. A. Cisneros). Recuperado de http://www.ualberta.ca/ iigm//pdfs/ introduccion.pdf

Modelo VARK (9 de agosto, 2013). [Mensaje en un blog]. Recuperado de https://maestriasutec. wordpress.com/3-3/ 
doi: http://dx.doi.org/10.15359/ree.20-3.10

URL: http://www.una.ac.cr/educare

CORREO: educare@una.cr

Porlán, R. (1987). El maestro como investigador en el aula. Investigar para conocer, conocer para enseñar". Investigación en la Escuela, 1, 63-69. Recuperado de http://www. investigacionenlaescuela.es/articulos/1/R1 9.pdf

Prado, M. (2004). Estilo de aprendizaje y rendimiento académico en un entorno virtual. Ponencia presentada en el II Congreso ONLINE OCS/ Ciber Sociedad. Recuperado de $\quad$ http://www.cibersociedad.net/congres2004/grups/fitxacom publica2. php?idioma $=$ es\&id $=492 \&$ grup $=29$

Rodríguez, M. (11 de agosto, 2009). Las TIC en la educación [mesaje en un blog]. Recuperado de http://ticsenlaeducacion-yaneth.blogspot.com

Torres, J. (1986). El diario escolar. Revista Cuadernos de Pedagogía, 142, 52-55.

Torres, G. M. y Redondo, A. C. y (2004). Tecnologías de información y comunicación. Conectividad y herramientas de trabajo colaborativo. Barranquilla: Publicaciones Universidad del Atlántico

Zapata-Ros, M. (2015). Teorías y modelos sobre el aprendizaje en entornos conectados y ubicuos. Bases para un nuevo modelo teórico a partir de una visión crítica del "conectivismo". Education in the Knowledge Society (EKS), 16(1), 69-102. doi: http://dx.doi.org/10.14201/ eks201516169102

\section{Cómo citar este artículo en APA:}

Acosta-Castillo, L. (Setiembre-diciembre, 2016). La relación entre los estilos de aprendizaje y el uso de las tecnologías de información y comunicación en educación de personas adultas. Revista Electrónica Educare, 20(3), 1-18. doi: http://dx.doi.org/10.15359/ree.20-3.10

Nota: Para citar este artículo en otros sistemas puede consultar el hipervínculo "Como citar el artículo" en la barra derecha de nuestro sitio web: http://www.revistas.una.ac.cr/index.php/EDUCARE/index 